\title{
EDITORIAL
}

\section{Delayed Activation of the Left Atrial Appendage Following Catheter Ablation of Persistent Atrial Fibrillation: A Cause for Concern?}

\author{
AMAN CHUGH, M.D. and HAKAN ORAL, M.D. \\ From the Division of Cardiovascular Medicine, University of Michigan, Ann Arbor, Michigan
}

\section{ablation, atrial fibrillation}

Despite advances in catheter ablation of patients with persistent atrial fibrillation (AF), the ideal ablation strategy still is in evolution. It is known, however, that these patients fare poorly with pulmonary vein (PV) isolation alone. ${ }^{1}$ In an effort to improve outcomes, potential mechanisms of AF beyond the PVs have been targeted by left atrial linear ablation, ${ }^{2,3}$ ablation of complex fractionated atrial electrograms (CFAEs), ${ }^{4}$ ablation of ganglionated plexi, and a combination thereof. Recently, "stepwise ablation," consisting of PV isolation, ablation of CFAEs, and linear ablation in an effort to terminate $\mathrm{AF}$, has been reported to be highly effective in patients with persistent $\mathrm{AF} .^{5}$ However, extensive ablation may be associated with impairment of left atrial (LA) contractility. Although sinus rhythm may have been attained, altered LA activation and mechanical function may lead to AV dyssynchrony and an ongoing risk of thromboembolic complications.

In this issue of PACE, Jiang et al. present their experience with extensive LA ablation in patients with persistent $\mathrm{AF}$ and resultant conduction delay into the left atrial appendage (LAA). ${ }^{6}$ Among 201 patients with persistent AF, LAA delay, defined as the activation of the LAA after the onset of the QRS complex on the surface electrocardiogram, was observed in 23 patients $(11 \%)$ after the first ablation procedure. The ablation strategy included PV isolation followed by linear ablation along the roof, LA septum (from the mitral annulus to the rightsided PVs or the roof line), mitral, and cavotricuspid isthmi. Delay in LAA activation developed in nine of the first 26 patients (35\%). Therefore, linear septal ablation was abandoned in favor of ablation of septal CFAEs in the remaining 175 patients. As a result, delay in LAA activation occurred in only five of these patients (3\%). LAA activation delay also developed in nine of 45 patients $(20 \%)$

Disclosures: Dr. Oral is a co-founder of Ablation Frontiers.

Address for reprints: Aman Chugh, M.D., Cardiovascular Center, SPC 5853, 1500 E. Medical Center Dr., Ann Arbor, MI 48109-5853. Fax: 734-936-7026; e-mail: achugh@umich.edu

doi: 10.1111/j.1540-8159.2010.02752.x who underwent a repeat procedure. Isolation of LAA was noted at the end of an index ablation procedure in one patient, and the atria were found to be dissociated in two patients after a repeat procedure.

In the majority of patients with LAA conduction delay, LA breakthrough during sinus rhythm occurred via the coronary sinus instead of the anterosuperior LA, indicative of Bachmann's bundle block (Fig. 1). In a subset of patients in whom an echocardiogram was performed during sinus rhythm prior to the ablation procedure, a significant decrease in mitral peak A-wave velocity was noted at 1 month after the procedure. In other patients with LAA conduction delay, mitral A-wave was absent. All patients with LAA delay remained on warfarin. Based on these observations, the authors advise caution during extensive ablation procedures, which may result in impaired LA transport function and increased risk of thromboembolic events.

Review of the LA myoarchitecture may be helpful to better understand how septal ablation may lead to the LAA conduction delay. The major inter- and intra atrial connections arise from the septal raphe or the interatrial groove. Epicardially, the leftward extension of Bachmann's bundle, located at the anterosuperior LA, joins with circular fibers of the anterior LA, which then bifurcates to embrace the ostium of the LAA. ${ }^{7}$ Deeper fibers of the septopulmonary bundle, which also arise from the septal raphe, and the septoatrial bundle, which are endocardial in location, combine to further reinforce the myocardium at the base of the LAA. ${ }^{8}$ Thus, it is possible to interrupt LAA conduction during radiofrequency (RF) energy delivery at the region of Bachmann's bundle,${ }^{9}$ the base of the LAA, or at any point in between. Indeed, a recent study demonstrated that the LAA may be isolated even when RF energy is delivered $>5 \mathrm{~cm}$ from the base of the LAA, underscoring the length of the intra-atrial connections. ${ }^{10}$

During stepwise ablation, RF energy delivery at the anterior aspect of the left-sided PVs, the LA septum and base of the LAA during CFAE ablation, and linear ablation at the mitral isthmus in

(C)2010, The Authors. Journal compilation (C)2010 Wiley Periodicals, Inc. 


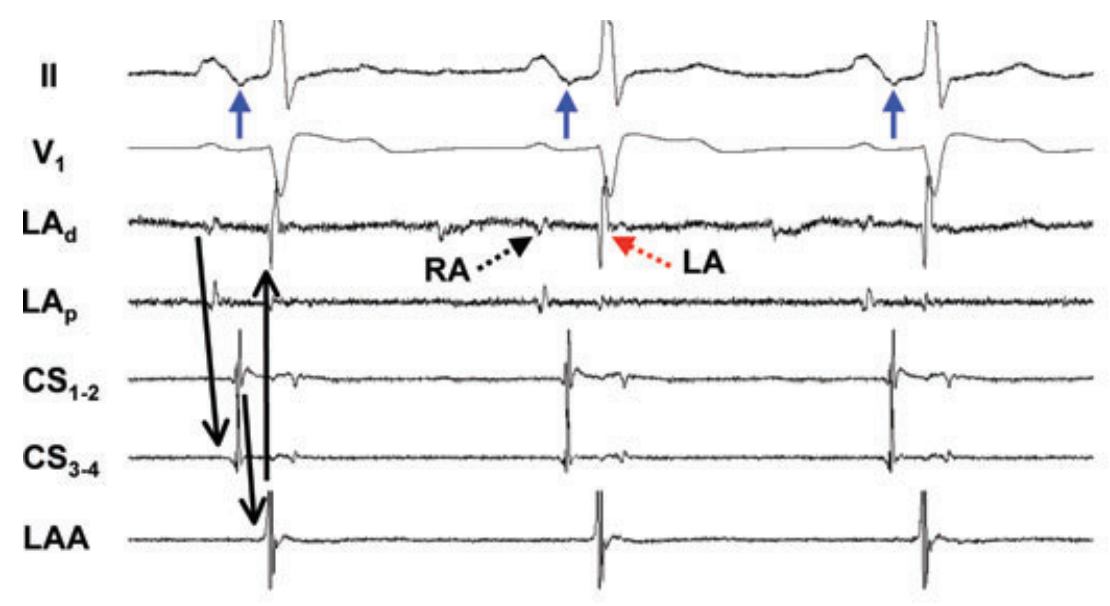

$500 \mathrm{~ms}$

Figure 1. Example of Bachmann's bundle block. This patient had previously undergone catheter ablation of persistent atrial fibrillation $(A F)$ and presented for a repeat procedure for atrial tachycardia. Linear ablation from the anterior aspect of the mitral valve to the anterosuperior left atrium (Bachmann's bundle region) terminated the tachycardia. During sinus rhythm, widely split double potentials could be recorded throughout the left atrial (LA) septum, consistent with dyssynchronous activation of the right (RA) and left atrium (LA). As a result, LA activation proceeds caudocranially via the coronary sinus, to the left atrial appendage (LAA), and then the LA septum (solid black arrows). Caudocranial activation of the LA results in the terminal negativity of the $P$ wave in the inferior leads (blue arrows). Also shown are electrocardiographic leads II and V1, bipolar electrograms recorded by the ablation catheter located at the LA septum, coronary sinus (CS), and the LAA.

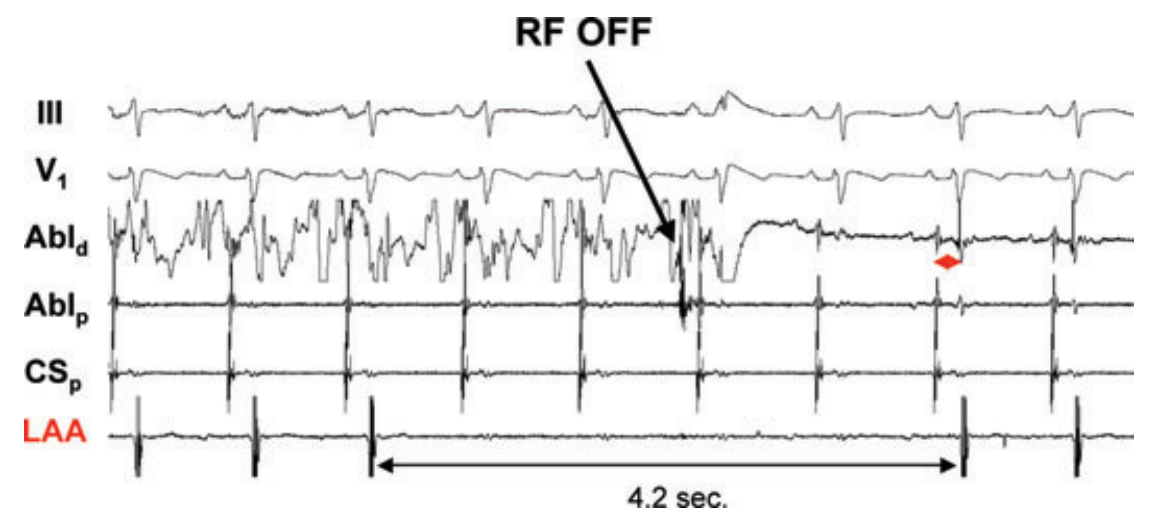

Figure 2. Isolation of the left atrial appendage (LAA). A repeat ablation procedure for atrial tachycardia (AT) was performed in a patient who previously had undergone LA ablation for chronic AF. After elimination of the AT, mapping during sinus rhythm revealed presence of fractionated electrograms along the anterior aspect of the left pulmonary vein antrum. Radiofrequency (RF) energy delivery for 8 seconds $(25 \mathrm{~W})$ at this site resulted in LAA isolation. Shortly after discontinuing RF delivery (arrow), LAA conduction recovered. Note that LAA activation occurred well after the onset of the QRS even prior to ablation, consistent with LAA delay. Also, note the presence of double potentials (red arrow) as a result of ablation, which were found throughout the base and apex of the LAA, indicative of conduction block. Annotations as above. 


\section{LA APPENDAGE DELAY FOLLOWING ABLATION}

essence transect the intra-atrial connections at various points. Although extensive ablation is likely an important determinant of LAA delay/isolation, the LAA may be isolated even with minimal ablation. A recent study showed that in patients with preexisting atrial scarring unrelated to the ablation procedure, RF energy delivery at critical sites may readily lead to LAA isolation. ${ }^{10}$

In the study by Jiang et al., LAA activation delay occurred in $\sim 11 \%$ of patients during stepwise ablation. Since LAA activation was not assessed prior to ablation, it is unclear whether there was any preexisting LAA activation delay, which would most likely be due to structural remodeling. Although delay is likely more common, LAA isolation is likely to be rare. Among $\sim 1,500$ patients undergoing catheter ablation of persistent AF or post-ablation atrial tachycardia at our institution, transient or permanent LAA isolation was observed in 11 patients $(<1 \%)$. The epicardial location of Bachmann's bundle and the redundancy provided by the deeper bundles may help explain why complete LAA isolation is rare.

There are several potential consequences of LAA activation delay, spontaneous or related to catheter ablation. First, atrial transport function may be impaired. It is important to bear in mind that patients with significant LAA activation delay frequently harbor areas of low voltage/scar throughout the LA. ${ }^{10}$ The widespread myopathy is likely to be associated with LA mechanical dysfunction. Second, electrical isolation of the LAA may result in stasis, which may promote thrombus formation, even during sinus rhythm. Although one may be willing to accept the trade off between sinus rhythm and delayed LAA conduction, this may not be entirely safe. Spontaneous LAA isolation, years after a prior procedure that demonstrated intact LAA conduction, has been

\section{References}

1. Oral H, Knight BP, Tada H, Ozaydin M, Chugh A, Hassan S, Scharf C, et al. Pulmonary vein isolation for paroxysmal and persistent atrial fibrillation. Circulation 2002; 105:1077-1081.

2. Haissaguerre M, Sanders P, Hocini M, Takahashi Y, Rotter M, Sacher F, Rostock T, et al. Catheter ablation of long-lasting persistent atrial fibrillation: Critical structures for termination. J Cardiovasc Electrophysiol 2005; 16:1125-1137.

3. Oral H, Pappone C, Chugh A, Good E, Bogun F, Pelosi F, Jr., Bates ER, et al. Circumferential pulmonary-vein ablation for chronic atrial fibrillation. N Engl J Med 2006; 354:934-941.

4. Nademanee K, McKenzie J, Kosar E, Schwab M, Sunsaneewitayakul B, Vasavakul T, Khunnawat C, et al. A new approach for catheter ablation of atrial fibrillation: Mapping of the electrophysiologic substrate. J Am Coll Cardiol 2004; 43:2044-2053.

5. Haissaguerre M, Hocini M, Sanders P, Sacher F, Rotter M, Takahashi Y, Rostock T, et al. Catheter ablation of long-lasting persistent atrial fibrillation: Clinical outcome and mechanisms of subsequent arrhythmias. J Cardiovasc Electrophysiol 2005; 16:1138-1147. described. ${ }^{10}$ Thus, we agree with the authors' recommendation that patients with significantly delayed LAA conduction should remain on warfarin long term until more data become available. The role of LAA occlusion devices in such patients is unknown. Third, marked delay in LAA activation may adversely affect hemodynamics such that LA contraction may occur too late, even after ventricular systole has commenced.

Due to the possibility of LAA activation delay and isolation, we monitor LAA conduction continuously in patients undergoing catheter ablation of persistent AF. After completion of PV isolation, the ring catheter is moved to the base of the LAA, which not only allows one to follow the AF cycle length but also to monitor the integrity of LAA conduction. During AF, RF energy delivery should be interrupted if there is evidence of entrance block into the LAA even when ablating several centimeters away from the appendage. During sinus rhythm, LAA conduction delay or isolation should prompt the immediate discontinuation of RF energy delivery, which typically results in resumption of conduction (Fig. 2). ${ }^{10}$ Another approach to prevent LA dysfunction and LAA isolation is to avoid extensive ablation along the long intra- and inter atrial conduction routes.

Stepwise ablation represents a major advance in the treatment of patients with persistent AF. However, the timely contribution by Jiang et al. reminds us that there may be unintended consequences of extensive ablation. Although the salutary effects of sinus rhythm even after extensive ablation are likely to be preferable to the detrimental consequences of untreated, persistent AF, the findings of the current study underscore the continuing need to explore novel techniques and modalities to further improve outcomes while minimizing unnecessary ablation.

6. Jiang C-X, Sang C-H, Dong J-Z, Liu X-P, Long D-Y, Yu R-H, Tang R-B, et al. Significant left atrial appendage activation delay complicating agressive septal ablation during catheter ablation of persistent atrial fibrillation. Pacing Clin Electrophysiol 2010; 33:652-660.

7. Ho S, Anderson R, Sanchez-Quintana D. Atrial structure and fibres: Morphological basis of atrial conduction. Cardiovasc Res 2002; 54:325-336.

8. Cabrera JA, Ho SY, Climent V, Sanchez-Quintana D. The architecture of the left lateral atrial wall: A particular anatomic region with implications for ablation of atrial fibrillation. Eur Heart J 2008; 29:356362 .

9. Kuhne M, Ho SY, Morady F, Chugh A. Elimination of left atrial appendage potentials during radiofrequency ablation near the right superior pulmonary vein. Heart Rhythm 2008; 5:475-478.

10. Chan C, Wong W, Pumprueg S, Veerareddy S, Billakanty S, Ellis $\mathrm{C}$, Chae S, et al. Inadvertent electrical isolation of the left atrial appendage during catheter ablation of persistent atrial fibrillation. Heart Rhythm 2010; 7:173-180. 